\section{Annals of Applied Microbiology \& Biotechnology Journal}

\section{Article Information}

Received date: Feb 12, 2019

Accepted date: Feb 25, 2019

Published date: Feb 27, 2019

\section{${ }^{*}$ Corresponding author}

Benard O Aliwa, Department of Food Science, Nutrition, and Technology, University of Nairobi, P. O. Box 29053-00625, Nairobi, Kenya; Mob: +254720345846; Email: bennkaliwa@ gmail.com (or) bochieng@uonbi.ac.ke

Distributed under Creative Commons CC-BY 4.0

Keywords Clostridium perfringens; Camel husbandry; Antibiotics resistance; Isiolo County; Kenya

Article DOI 10.36876/aamb.1012

\section{Antibiotic Resistance of Clostridium Perfringens Isolated from Raw Camel Milk in Isiolo County, Kenya}

\author{
Benard O Aliwa* and Kaindi Dasel Wambua Mulwa \\ Department of Food Science, Nutrition, and Technology, University of Nairobi, P. O. Box 29053-00625, \\ Nairobi, Kenya
}

\section{Abstract}

Clostridium perfringens is a gram-positive, anaerobic, motile rod spore forming bacteria. It causes food poisoning and spoilages in milk and milk products. The camel husbandry and health management practices in the Arid and Semi-Arid Lands (ASALs) of Kenya increase the possibility of contamination, transmission, and development of antibiotic resistance zoonotic organisms. Therefore, the objective of this study was to estimate the prevalence of antibiotic resistant $\mathrm{Cl}$. perfringens strains in camel milk in Isiolo County, Kenya. A total of 308 raw camel milk were aseptically harvested from randomly selected camels from 15 herds along selected camel milk clusters in Isiolo County. $48.05 \%$ (148/308) of all the sampled camel milk were positive for Clostridium species and out of the 148 samples that were positive for Clostridium species, $19.1 \%$ (59/148) were positive for $\mathrm{Cl}$. perfringens. Out of the 59 isolates that were confirmed as $\mathrm{Cl}$. perfringens, the isolates that were resistant to tested antibiotics, in decreasing order, were ampicillin $(61.02 \%)$, Sulphamethazole $(47.46 \%)$, Cotrimazole $(45.76 \%)$, Streptomycin $(44.07 \%)$, Chloramphenicol $(42.37 \%)$, Kanamycin $(40.68 \%)$, Tetracycline $(37.29 \%)$, and Gentamycin (35.59\%). Therefore, these findings established contamination of raw camel milk with Cl. perfringens characterized with antibiotics resistance capabilities posing a health risk to the local communities that consume the unpasteurized camel milk.

\section{Introduction}

Clostridium perfringens, a gram-positive anaerobic rod spore forming bacteria, spores are ubiquitous in unhygienic farm environment including soil, dust, water, sewage, sediments and vegetation [1-2]. Animals are readily exposed to $\mathrm{Cl}$. perfringens in farm and grazing environment; from where they enter the gastrointestinal tract (GIT) and other body tissues and rapidly proliferate and cause zoonotic diseases. The camel husbandry and health management practices in the Arid and Semi-Arid Lands (ASALs) of Kenya increase the possibility of contamination, transmission, and development of antibiotic resistance zoonotic organisms.

The development of antimicrobial resistant microorganisms results from misuse, overuse, under/ inadequate use of antimicrobials, animal health practices common in ASALs of Kenya. This is due to inadequate provision of veterinary services leading to self-medication of camels [3-4]. The ASALs' climate and unhygienic environment like erratic rainfall, leading to inadequate grazing pastures and shrubs, inadequate and unhygienic animal watering resources, and dusty grazing, watering and housing environment makes camel husbandry management practices conducive for development of antimicrobial resistance and spread of zoonotic microorganisms. Therefore, the WHO Global Strategy and intervention framework of containment of antimicrobial resistance through slowing down emergence and reducing the spread of antimicrobial resistant microorganisms is difficult to attain in ASALs.

It has been reported that zoonotic pathogens, like spore-former $\mathrm{Cl}$. perfringens, are more sensitive to climate change than human- or animal-only pathogens [5], suggesting that zoonotic pathogens are likely to be impacted more seriously by climate change. Some climate change drivers like erratic moisture and rainfall, and high temperatures are frequently found in the ASALs, hence likely to facilitate high mutation rate, horizontal gene transfer, development of antimicrobial resistant and transmission of food, water- and soil-borne resistant pathogens [6].

Previously, camels were considered resistant to most of the diseases commonly affecting livestock, but current research indicates that camels are susceptible to a large number of pathogenic agents [7]. Camel calf diarrhea and high camel calf and herd morbidity and mortality rate has been reported by veterinary researchers and pastoralist in Kenya as being the common disease affecting suckling dromedary calves, with serious effects on herd growth. This can be attributed to late veterinary intervention, a common case in ASALs due to inaccessible of veterinary services to where camels are kept [4]. Camel calf diarrhea is caused by mixed microbial infection, notably Salmonella spp., Escherichia coli and Clostridium perfringens[8]. There is high chance of this

\title{
ISSN: $2576-5426$
}


causative organisms being secreted in camel milk and causing milkborne infection to human. Under harsh ASALs climatic condition $\mathrm{Cl}$. perfringens being a spore-former can undergo mutation leading to development antibiotic resistant strains that can under the unhygienic milk production and harvesting condition contaminate camel milk and subsequently consumers, hence public health concern. However, there is no information and data on prevalence of spore-forming and antibiotic resistant $\mathrm{Cl}$. perfringens in camel milk in Kenya. Therefore the objective of this study was to estimate the prevalence of antibiotic resistant $\mathrm{Cl}$. perfringens strains in camel milk in Isiolo County, Kenya.

\section{Materials and Methods}

\section{Study Site}

The study was carried out along the Mlango-Ngarendare-Burat, KambiGarba-Ngaremara-Chumvi-Gambela, and Boji-KulamaweBaranbate camel milk clusters in Isiolo County (Figure 1). Isiolo County is a typical arid and semi-arid lands (ASALs) area located in Northern eastern region of Kenya. The County has camel population of approximately 43,300 camels, kept under peri-urban and pastoral camel production systems. The Isiolo County has a thriving camel milk production and marketing business. It supplies $90 \%$ of camel milk to the Nairobi terminal camel milk market.

\section{Sample Collection and Preparation}

Raw camel milk was aseptically harvested from 308 camels randomly selected from 15 camel herds along selected camel milk clusters in Isiolo County, Kenya. Immediately after harvesting, the milk samples were kept in ice box containing ice packs and transported toIsiolo County Veterinary Office laboratory. At the County Veterinary Office laboratory the raw camel milk samples were kept in deep freezer maintained at $-20^{\circ} \mathrm{C}$ until transported to Molecular Laboratory, at the Department of Food Science, Nutrition and Technology, University of Nairobi and stored in deep freezer maintained at $-50^{\circ} \mathrm{C}$.

\section{Isolation and Identification of Clostridium perfringens species}

Frozen raw camel milk samples were thawed overnight at room temperature. Isolation of Clostridium species was performed by streaking camel milk samples on Reinforced Clostridium agar (Blalab, Merck Ltd, South Africa) with added egg yolk emulsion and incubated anaerobically at $37^{\circ} \mathrm{C}$ for $24-48 \mathrm{hrs}$. The typical $\mathrm{Cl}$. perfringens isolates were identified on the basis of colony morphology, motility, and $\beta$ hemolysis reaction [9-11]. Characteristic Cl. perfringens isolates were black in colour, surrounded with clear zone, large and flat with wide spreading, irregular, coarse rhizoid margin and raised centre. The clear opaque zone surrounding colonies indicate lecithinase activity. $\mathrm{Cl}$. perfringens is non-motile and normally grows at $44^{\circ} \mathrm{C}$, whereas some other clostridia are inhibited at this temperature. $\mathrm{Cl}$. perfringens is non-motile and normally grows at $44^{\circ} \mathrm{C}$, whereas some other clostridia are inhibited at this temperature.

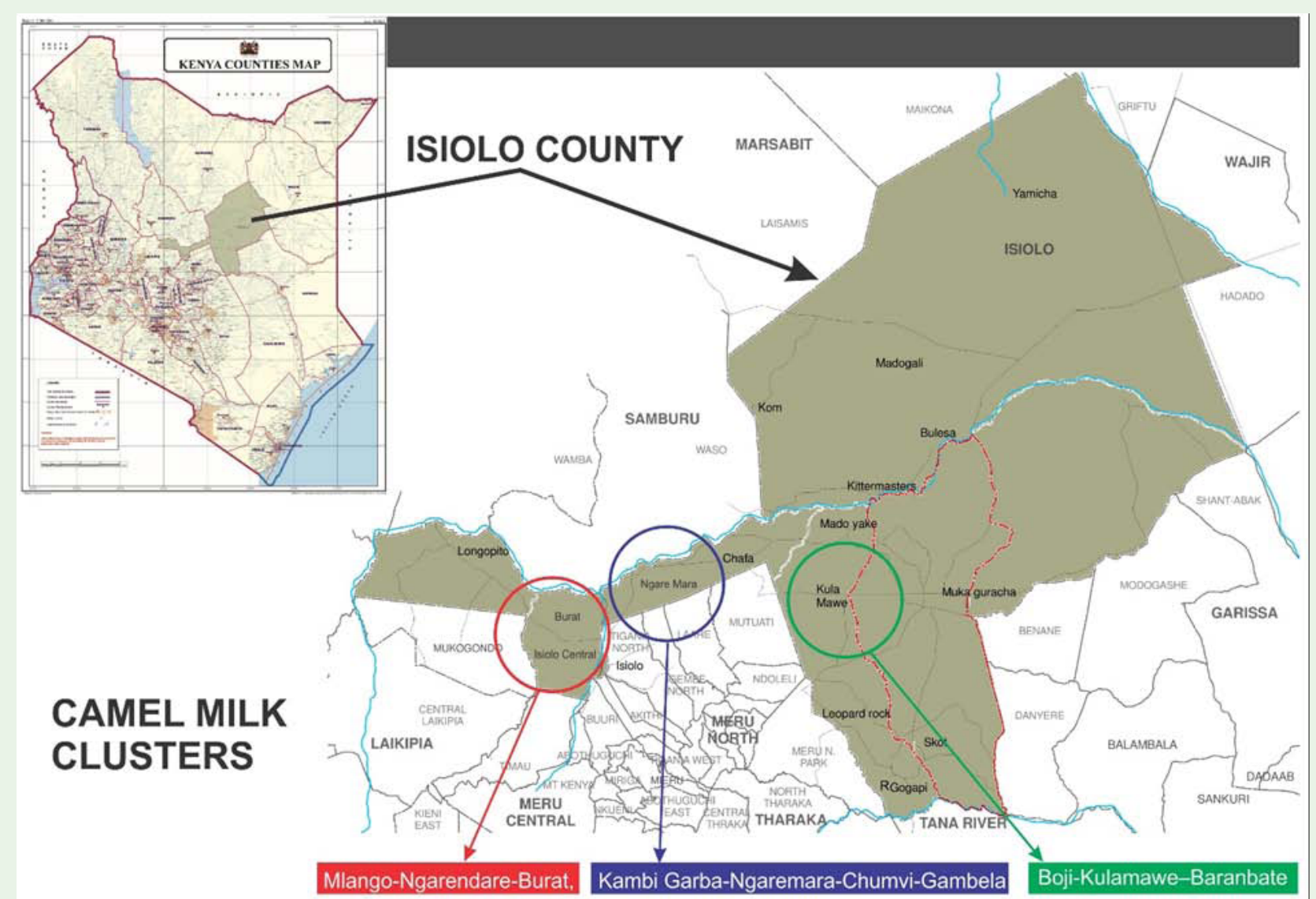

Figure 1: Map of ASALs Isiolo County showing camel milk marketing clusters. 


\section{Motility test}

Suspected Clostridium isolates were inoculate by stabbing into the center of the Sulfide-Indole-Motility (SIM) Medium, a semisolid that allow for the detection of bacterial motility, and incubate at $35 \pm 2^{\circ} \mathrm{C}$ for $24-48$ hours. Motility was demonstrated by growth away from the stab spot and turbidity or cloudiness throughout the medium. Non-motile organisms grow only along the stab spot and leave the surrounding medium clear $\mathrm{Cl}$. perfringens is non-motile.

\section{Blood agar for hemolytic activity}

Suspected Clostridium isolates were spread on horse blood agar media and incubated anaerobically for 24 hours at $37^{\circ} \mathrm{C}$. The Blood agar plates were examined for round, smooth, circular, gray white colour colonies surrounded by a typical zone of hemolysis ( $\beta$-hemolysis) which is characteristics of $\mathrm{Cl}$. perfringens produces large beta-hemolytic colonieson Blood Agar, but some $\mathrm{Cl}$. perfringens strains produce a double zone of hemolysis.

\section{Determination of antibiotic resistance among Clostridium perfringens isolates}

Antibiotic resistance testing was performed by the Kirby-Bauer disc diffusion method using Mueller-Hinton (MH) agar. The typical $\mathrm{Cl}$. perfringens isolates were cultured overnight in Trypticase-soy broth (TSB) supplemented with $0.6 \%$ yeast extract. Approximately
$0.1 \mathrm{ml}$ of broth culture was spread plated onto $\mathrm{MH}$ agar, diffusion KGL 2/4 octodiscs containing of antibiotics namely: - Ampicillin $(25 \mu \mathrm{g}$, Tetracycline $(25 \mu \mathrm{g})$, Cotrimazole $(25 \mu \mathrm{g})$, Streptomycin $(10 \mu \mathrm{g})$, Kanamycin $(30 \mu \mathrm{g})$, Gentamicin $(10 \mu \mathrm{g})$, Sulphamethazole $(200 \mu \mathrm{g})$ and Chloramphenicol $(30 \mu \mathrm{g})$ (Himedia) introduce onto plates and plates incubated at $37^{\circ} \mathrm{C}$ in $5 \% \mathrm{CO}_{2}$ for 18 hours. Octodiscs are flat circular ring having 8 discs coated with antibiotics that aid in determination of multi-antibiotic resistance organisms (Figure 2). The plates were examined for growth, clear zone surrounding the antibiotic disc and no clearing surrounding the antibiotic disc. No clearing surrounding the antibiotic disc indicated antibiotic resistance.

\section{Results}

Out of 308 samples of raw camel milk examined 48.05\% (148/308) were positive for Clostridium species. Out of 148 samples of raw camel milk that showed presence of Clostridium species, $19.1 \%$ (59/308) samples showed presence of $\beta$-hemolysis on blood agar and non-motility on SIM agar, indicating presence of Cl. Perfringens. Out of the 59 isolates that were positive for $\mathrm{Cl}$. perfringens, the isolates that were resistant to tested antibiotics, in decreasing order, were ampicillin (61.02\%), Sulphamethazole (47.46\%), Cotrimazole (45.76\%), Streptomycin (44.07\%), Chloramphenicol (42.37\%), Kanamycin (40.68\%), Tetracycline (37.29\%), and Gentamycin (35.59\%) (Table 1).

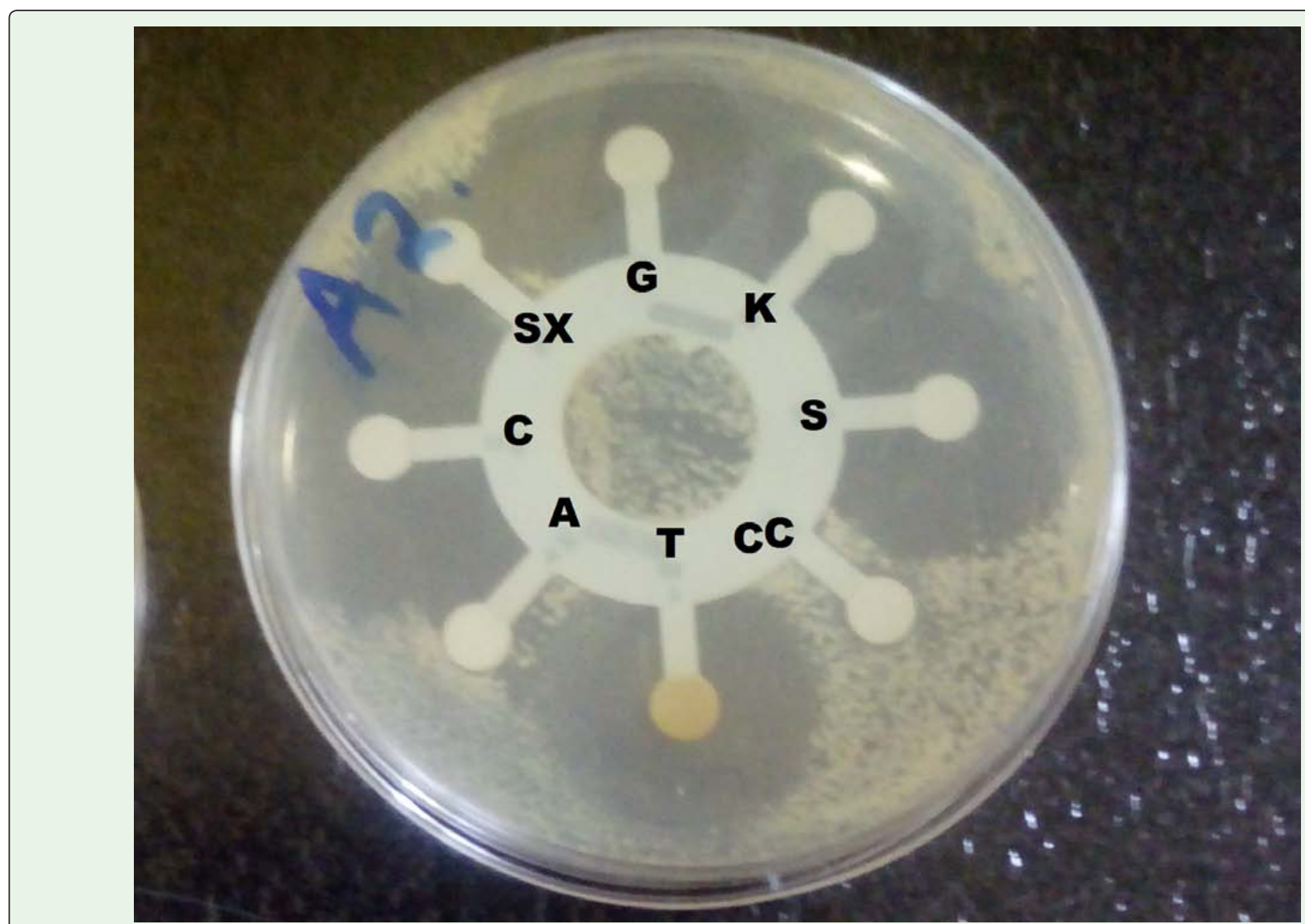

Figure 2: Showing multiple antibiotic resistance of Clostridium perfringens isolate to various antibiotics. Ampicillin (A), Tetracycline (T), Cotrimazole (CC), Streptomycin (S), Kanamycin (K), Gentamicin (G), Sulphamethazole (Sx), Chloramphenicol (C). 
Table 1: Antibiotic resistance (expressed as \%) of Clostridium perfringens isolated from camel milk in Isiolo County, Kenya.

\begin{tabular}{|l|c|c|c|}
\hline & Antibiotic & Number Resistant Isolate (n=59) & Percentage of Resistant Isolates \\
\hline 1 & Ampicillin & 36 & 61.02 \\
\hline 2 & Tetracycline & 22 & 37.29 \\
\hline 3 & Cotrimazole & 27 & 45.76 \\
\hline 4 & Streptomycin & 26 & 44.07 \\
\hline 5 & Kanamycin & 24 & 40.68 \\
\hline 6 & Gentamicin & 21 & 35.59 \\
\hline 7 & Sulphamethazole & 28 & 47.46 \\
\hline 8 & Chloramphenicol & 25 & 42.37 \\
\hline
\end{tabular}

\section{Discussion}

This study demonstrates presence of multi-drug resistant of $\mathrm{Cl}$. perfringens isolates in camel milk in the study area. Treatment of animal requires antimicrobial agents like antibiotics, however, improper use of this antimicrobial agents can results in development of antimicrobial resistance. The high resistance to antibiotic could be attributed to easy availability of antibiotics over the counter and misuse, overuse, under/inadequate use of antibiotic during treatment of camels by pastoralists' [4]. The antibiotic resistant $\mathrm{Cl}$. perfringens can contaminate raw camel milk either through animal faecal contamination, unhygienic harvesting, and handling or from dusty environmental sources [12-13]. From this study it was established that $48.05 \%(148 / 308)$ of the analyzed milk samples were positive for Clostridium species. In a study conducted by [9], the findings revealed that $52 \%$ of the raw milk obtained from dairy animals was contaminated with Clostridium species. The small difference between the two findings can be related to difference in sample size used in the two studies and also type of the samples used. The occurrence of Clostridium species in milk products is hazardous posing a serious health risk to milk consumers, especially in the absence of pasteurization or sufficient boiling. Therefore, these findings underscore the need to enhance milk handling hygiene and improved policy on antibiotic use in treatment of camels.

The easy availability of antibiotics over the counter, despite regulations to the contrary, contributes to increased inappropriate usage of antimicrobial drugs in Kenya. Resistance to antimicrobial agents (AMR) result in treatment failures and increased health care costs, hence emergent antibiotic resistance is a serious public health risk and global problem. (49.2\%) of the $\mathrm{Cl}$. Perfringens isolates in this study showed multi drug resistance to the antibiotics. Majority, 39 out of $59(61 \%)$ of the isolates were resistant to Ampicillin while minority (35\%) being resistant to gentamycin. The rates of antibiotic resistance differ significantly depending on geographical locations and the existing national policy on the use of antibiotics (14). For example, various studies have established highest level of $\mathrm{Cl}$. Perfringens resistance to clindamycin and cephalosporins (55\% and 51\%, respectively). Most of the isolates were susceptible to aminoglycosides including gentamycin, kanamycin, and streptomycin $(65 \%, 60 \%$, and $56 \%$, respectively). $37 \%$ of the $\mathrm{Cl}$. Perfringens isolates were resistant to Tetracycline based on the findings from this study this was in agreement with findings by (15), whereby it was established $20 \%$ to $50 \%$ of the $\mathrm{Cl}$. Perfringens isolates were resistant against tetracycline. However, the isolates were obtained from different samples. From this study the findings revealed that $42 \%$ of the $\mathrm{Cl}$. Perfringens isolate were resistant to chloramphenicol this is contrary to the findings obtained by [16], whereby $46 \%$ of the $\mathrm{Cl}$. Perfringens were resistant to chloramphenicol. This difference in the findings can be attributed to the fact that the two researches were conducted in different geographical setting one in Kenya and the other in Egypt. According to According to [16], the rates of antibiotic resistance differ significantly depending on geographical locations and the existing national policy on the use of antibiotics.

\section{Conclusion}

An extensive resistance to multiple antibiotics was detected in $C$. perfringens isolates from camel milk in this study. Therefore, episodic monitoring of trends in Antibiotic resistance patterns is logical, since there is possibility of $C$. perfringens being a source of resistance genes transferring to other species of bacteria that are found in the camel milk. There is significant potential for therapeutic challenges in the future unless care is taken to avoid the selection of multi-resistant organisms.

\section{Acknowledgments}

The authors of would like to sincerely thank the technical staff in the molecular microbiology laboratory of the University of Nairobi, Department Food Science Nutrition and Technology for their support.

\section{References}

1. Coorevits A, Jonghe VD, Vandroemme J, Reekmans R, Heyrman J, Messens $\mathrm{W}$, et al. Comparative analysis of the diversity of aerobic spore-forming bacteria in raw milk from organic and conventional dairy farms. Syst Appl Microbiol. 2008; 31: 126-140.

2. Del Torre M, Stecchini ML, Braconnier A, Peck MW. Prevalence of Clostridium species and behaviour of Clostridium botulinum in gnocchi, a REPFED of Italian origin. Int J Food microbial. 2004; 96: 115-131.

3. Abdurahman OAS. Milk and meat from the camel: handbook on products and processing. vdfHochschulverlag AG. 2004.

4. Lamuka PO, Njeruh FM, Gitao GC, Abey KA. Camel health management and pastoralists' knowledge and information on zoonoses and food safety risks in Isiolo County, Kenya. Pastoralism. 2017; 7: 20-30.

5. Songer JG. Clostridia as agents of zoonotic disease. Vet Microbiol. 2010: 140: 399-404.

6. McIntyre KM, Setzkorn C, Hepworth PJ, Morand S, Morse AP, Baylis M. Systematic assessment of the climate sensitivity of important human and domestic animals pathogens in Europe. Sci Rep. 2017; 7: 7134. 
7. Abbas B, Agab H. A review of camel brucellosis. Prev Vet Med. 2002; 55 : 47-56.

8. Bengoumi M, Berrada J, Rochdi M, Hidane K, Lafarge FD, Faye B INFECTIOUS DISEASES-Physiopathology of diarrhea in the camel calf in Morocco. Clinical signs and metabolic disturbances. Revue d'Elevageet de MedecineVeterinaire des Pays Tropicaux. 1998; 51: 277-281.

9. Chaturvedi A, Shukla S. Occurrence of Clostridium species in different dairy products and its associated health risk. Intern J Recent Sci Res. 2015; 6 : 2827-2829.

10. Garde S, Arias R, Gaya, P, Nuñez M. Occurrence of Clostridium spp. in ovine milk and Manchego cheese with late blowing defect: identification and characterization of isolates. International dairy journal. $2011 ; 21: 272-278$.

11. Murray PR, Baron EJO, Jorgensen JH, Pfaller MA, Yolken RH. Manual of clinical microbiology. 2003; Volume 1, $8^{\text {th }}$ Edition, ASM Press, Washington, DC.
12. Odongo NO, Lamuka PO, Matofari JW, Abong GO. Risk factors associated with the post-harvest loss of milk along camel milk value chain in Isiolo County, Kenya. African Journal of Agricultural Research. 2016; 11: 674-682.

13. Wafula WN, Matofari WJ, Nduko MJ, Lamuka P. Effectiveness of the sanitation regimes used by dairy actors to control microbial contamination of plastic jerry cans' surfaces. International Journal of Food Contamination. 2016; 3: 9 .

14. Spigaglia $P$. Recent advances in the understanding of antibiotic resistance in Clostridium difficile infection. Ther Adv Infect Dis. 2016; 3: 23-42.

15. Kouassi KA, Dadie AT, N'Guessan KF, Dje KM, Loukou YG. Clostridium perfringens and Clostridium difficile in cooked beef sold in Cote d'Ivoire and their antimicrobial susceptibility. Anaerobe. 2014; 28: 90-94.

16. Osman KM, Elhariri M. Antibiotic resistance of Clostridium perfringens isolates from broiler chickens in Egypt. Rev Sci Tech. 2013; 32: 841-850. 